\title{
Homocysteine concentrations in the cognitive progression of
}

Alzheimer's disease

RUNNING HEADER: Homocysteine and cognition in Alzheimer's disease

Nicolas Farina ${ }^{\mathrm{a}^{*}}$, Fredrik Jernerén ${ }^{\mathrm{b}, 1}$, Cheryl Turner ${ }^{\mathrm{b}}$, Kathryn Hart $^{\mathrm{c}} \&$ Naji Tabet $^{\mathrm{a}, \mathrm{d}}$.

${ }^{\mathrm{a}}$ Centre for Dementia Studies, Brighton and Sussex Medical School, Brighton, BN19RY, UK.

bDepartment of Pharmacology, University of Oxford, Oxford, OX1 3QT, UK

'Department of Nutritional Sciences, University of Surrey, Guildford, GU27XH, UK

${ }^{\mathrm{d} D e m e n t i a}$ Research Unit, Sussex Partnership NHS Foundation Trust, Crowborough, TN6 1HB, UK

*Corresponding Author: Nicolas Farina, Centre for Dementia Studies, Brighton and Sussex Medical School, UK. BN1 9RY. N.Farina@ bsms.ac.uk. +441273678995

${ }^{1}$ Present address: Department of Pharmaceutical Biosciences, Uppsala University, 75237 Uppsala, Sweden.

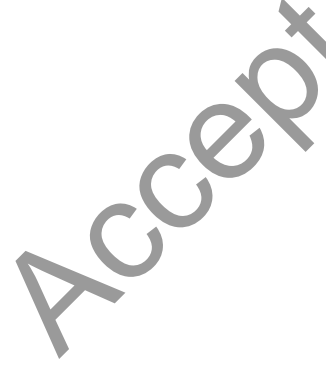

Please cite as: Farina,. N., Jenerén, F., Turner, C., Hart, K., \& Tabet, N. (2017) Homocysteine concentrations in the cognitive progression of Alzheimer's disease. Experimental Gerontology.

(C) 2017. This manuscript version is made available under the CC-BY-NC-ND 4.0 license http://creativecommons.org/licenses/by-nc-nd/4.0/ 


\section{Abstract:}

Objectives: Hyperhomocysteinemia in Alzheimer's disease (AD) is widely reported and appears to worsen as the disease progresses. While active dietary intervention with vitamins B12 and folate decreases homocysteine blood levels, with promising clinical outcomes in Mild Cognitive Impairment (MCI), this so far has not been replicated in established AD populations. The aim of the study is to explore the relationship between hyperhomocystenemia and relevant vitamins as the disease progresses.

Methods: In this longitudinal cohort study, 38 participants with mild to moderate AD were followed for an average period of 13 months. Plasma folate, vitamin B12 and homocysteine concentrations were measured at baseline and at follow-up. Dietary intake of B vitamins was also measured. Spearman's correlations were conducted by homocysteine and B vitamin status.

Results: As expected, cognitive status significantly declined over the follow-up period and this was paralleled by a significant increase in homocysteine concentrations $(p=0.006)$. However, during this follow-up period there was no significant decline in neither dietary intake, nor the corresponding blood concentrations of vitamin B12/folate, with both remaining within normal values. Changes in blood concentrations of B vitamins were not associated with changes in homocysteine levels ( $p>0.05)$.

Conclusion: In this study, the increase in homocysteine observed in AD patients as the disease progresses cannot be solely explained by dietary and blood levels of folate and vitamin B12. Other dietary and non-dietary factors may contribute to hyperhomocysteinemia and its toxic effect in $\mathrm{AD}$, which needs to be explored to optimise timely intervention strategies. 
Keywords: homocysteine, B vitamins, Alzheimer's disease, cognition

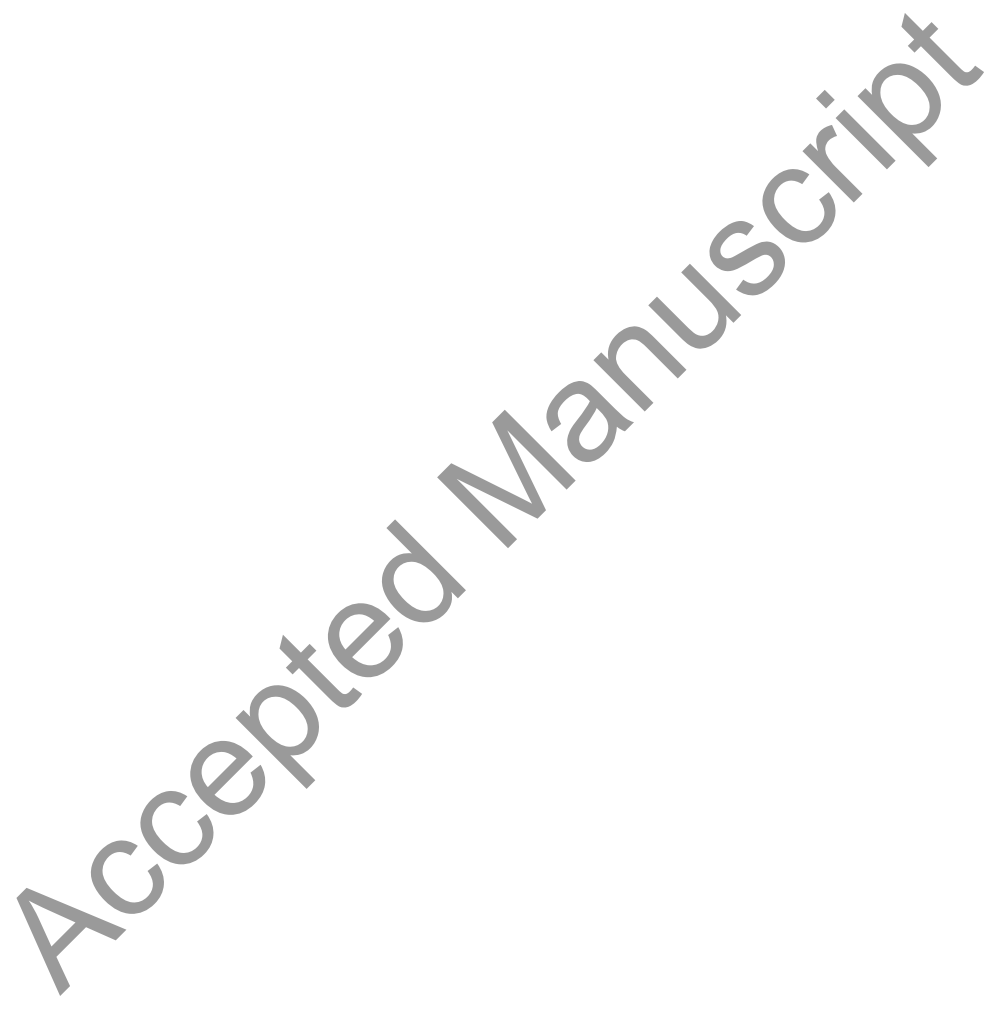




\section{Introduction}

Dementia is a common disorder with its prevalence set to rise worldwide. In the absence of a cure there is a growing need to identify treatments that can slow the cognitive progression of the disease, thus enabling those with dementia to retain appropriate functional abilities for longer. Currently, there are only a small number of symptomatic medications licensed for the treatment of Alzheimer's disease (AD), the most prevalent type of dementia. Importantly, the effectiveness of these licensed medications are often considered limited (Casey et al., 2010). Abnormal elevation of homocysteine levels has been implicated as a marker for AD. Hyperhomocysteinemia is associated with increased cognitive decline in healthy older adults with higher risk of cognitive impairment (e.g. (Morris, 2012; Smith et al., 2010; Vogel et al., 2009). Apart from a few notable exceptions, most studies report mild to moderate hyperhomocysteinemia in dementia populations compared to healthy controls (for a review see (Zhuo et al., 2011). However, research into the specific role of homocysteine on cognitive decline in a population with established AD is limited. Although not consistently reported (e.g. (Huang et al., 2010), studies have found that homocysteine levels in AD can predict rates of cognitive decline (Huang et al., 2013; Oulhaj et al., 2010).

It has therefore been of interest to establish whether reducing homocysteine levels can have a beneficial effect on the slowing down of AD progression. A key avenue of research is the supplementation with vitamin B12 and folate. These vitamins act as cofactors for the methylation of homocysteine to methionine, and therefore in the absence of these dietary nutrients homocysteine concentrations increase. It has been estimated that $65 \%$ of the hyperhomocysteinemia cases among the Framingham elderly population-based cohort could be accounted for by inadequate folate, or, to a lesser extent, inadequate vitamin B12 or 
vitamin B6 status and intake (Selhub et al., 1993). It has even been shown that B vitamins taken at the earliest stages of the disease (i.e. Mild Cognitive Impairment (MCI)) are able to slow down the rate of brain atrophy (Smith et al., 2010) as well as the cognitive decline (de Jager et al., 2012), and may reduce conversion rates into dementia (Blasko et al., 2013). Despite this, there is little evidence that increasing the intake of these vitamins are able to combat cognitive decline later in the disease course, i.e. among participants with established AD. Serum levels of vitamin B12 have been found to have no association with cognitive progression in AD populations (Huang et al., 2010; Oulhaj et al., 2010; Small et al., 1997a, 1997b; Tu et al., 2010). Randomised controlled trials (RCTs) in AD populations have found that relevant vitamin treatments are unable to attenuate cognitive progression in the samples as a whole (Aisen et al., 2008; Kwok et al., 2011; Sun et al., 2007). Interrogation of these findings suggest that B vitamin supplementation may still have a role in attenuating cognitive decline in $\mathrm{AD}$ only in the presence of particularly elevated homocysteine levels. For example, Kwok and colleagues found that participants who received B vitamin treatment with elevated levels of homocysteine ( $13 \mu \mathrm{mol} / \mathrm{L}$ or greater) had significantly smaller decline in the construction domain of Mattis Dementia Rating Scale (MDRS) than those receiving placebo (Kwok et al., 2011). Interestingly, participants in another study that had more modest levels of homocysteine $($ mean $=9.2 \mu \mathrm{mol} / \mathrm{L}, \mathrm{SD}=3.2$ ) at the onset of the trial did not report any positive effects on cognition, while a subgroup with mild AD showed some benefit of the treatment (Aisen et al., 2008).

Discerning the exact role of homocysteine and B vitamins on cognitive decline is complicated, as hyperhomocysteinemia is only one of many contributing markers in the disease progression. The neurotoxicity of homocysteine and its direct effect on brain atrophy has been established (Madsen et al., 2015). Unlike the early indications from MCI, lowering homocysteine levels in established AD may not produce the same clinical benefits as research 
so far has shown. Other non-dietary factors may also contribute to hyperhomocysteinemia and moderate its toxicity. Longitudinal studies assessing the relationship between homocysteine concentrations and cognitive decline in established AD, and its possible association with both dietary and blood vitamin B12 and folate levels, are limited (Clarke et al., 1998; Oulhaj et al., 2010).

The aim of this study was to establish whether the extensively reported hyperhomocysteinemia in $\mathrm{AD}$ as the disease progresses is associated with dietary and blood levels of B vitamins. In line with current knowledge, we hypothesised that blood homocysteine levels would significantly increase over the follow-up period. In addition, we hypothesised that this increase in homocysteine is explained by an âssociated decrease in vitamin B levels. 


\section{Materials and Methods}

\subsection{Participants}

All participants were recruited in Sussex (UK) memory assessment clinics and had mild to moderate $\mathrm{AD}(\mathrm{MMSE}>12)$. The mean age of $\mathrm{AD}$ participants was 81.3 years $(\mathrm{SD}=6.0)$. The inclusion and exclusion criteria for this study has previously been reported elsewhere (Farina et al., 2016). In brief, eligible participants had previously been clinically assessed using the International Statistical Classification of Diseases, 10th revision (ICD-10; World Health Organisation, 1992), and received a diagnosis of probable dementia of Alzheimer's type. AD patients all had a personal consultee (relative or friend), and were either clinically or selfreferred. For inclusion in the study described here, participants were also required to provide a blood sample on two occasions so that homocysteine concentrations could be measured. Ethical approval was obtained from a National Research Ethics Service Committee.

\subsection{Neuropsychological testing and dietary data}

Demographic data including age, gender and ethnicity were recorded. As previously reported (see Farina, Tabet, \& Rusted, 2016), a battery of neuropsychological tests and the Food Frequency Questionnaire (FFQ) were completed by participants and lasted approximately 2-3 hours. The Addenbrooke's Cognitive Examination Revised (ACE-R; Mioshi et al., 2006) was used to provide a standardised measure of dementia severity. The ACE-R total score was also used as a comprehensive measure of global cognitive status. The Cornell Scale of Depression in Dementia (CSDD; Alexopoulos et al., 1988) was used to screen for the potential presence of major depression. IQ was estimated using the National Adult Reading Test (NART; Nelson, 1982). 
Dietary intake of nutrients were estimated using the EPIC FFQ (Bingham et al., 2007). The questionnaire was completed by the carer of the participant, who described the average dietary intake of the participant over the past year. Once collected, data was analysed by the Department of Nutritional Sciences, University of Surrey, UK. Nutritional values for individual foods were estimated and summed. Nutrient intakes were expressed as percentages of the age and gender appropriate Recommended Nutritional Intakes (RNIs).

\subsection{Biochemical assays}

Plasma homocysteine, vitamin B12, and folate were analysed at the Department of Pharmacology, University of Oxford. Plasma concentrations of total Cobalamin (B12) and folate were measured by automated (Perkin-Elmer MûltiProbe 11 liquid handling system, Perkin-Elmer Life and Analytical Sciences) microbiological assays using Lactobacillus leichmanii and L. casei, respectively (Kelleher and Broin, 1991; Molloy and Scott, 1997). Between-day coefficients of variation for $\mathrm{B} 12$ were $7.1 \%$, and $7.4 \%$ for folate. Total plasma homocysteine was analysed byliquid-chromatography according to a modified protocol described previously (Refsum et al., 2004), using a QTRAP 5500 (AB Sciex, Framingham, Massachusetts, US) coupled to a Prominence LC-20ADxR binary pump (Shimadzu, Kyoto, Japan). In short, $10 \mu \mathrm{L}$ of plasma was added to an equal volume of an internal standard mix containing isotopically labelled homocysteine. Samples were neutralised using an ammonia solution before reduction with dithioerythriol at room temperature for $15 \mathrm{~min}$. Plasma proteins were precipitated with perchloric acid (4\% v/v) and cleared by centrifugation. Supernatant was diluted 1:10 in water/sodium 1-heptane sulfonate $[1 \mathrm{M}] /$ perchloric acid [20\%, $3.3 \mathrm{M}]$ 5/3/1 v/v/v, and injected onto a Kinetex C18 column $(30$ x $4.6 \mathrm{~mm}, 2.6 \mu \mathrm{m}$, Phenomenex, Torrance, CA, US) at a flow rate of $0.8 \mathrm{~mL} / \mathrm{min}$. Gradient elution was 
employed starting at $100 \%$ mobile phase A (water $+0.05 \%$ formic acid), with a final composition of $40 \% \mathrm{~A}$ and $60 \%$ mobile phase B (methanol $+0.05 \%$ formic acid). Data acquisition and analysis were performed with Analyst 1.6.1 (AB Sciex, Framingham, Massachusetts, US). Quantitation was based on the ratio of analyte peak area/internal standard peak area against a linear calibration curve with a 1/x weighting. The coefficient of variation for was $4.0 \%$.

\subsection{Statistical Analysis}

Descriptive statistics (e.g. means and frequencies) were reported for the participant demographics. All findings reported were of participants that were able to provide a sample of blood at baseline and at follow-up so that plasma homocysteine concentrations could be analysed. A series of Spearman's Rho correlations were conducted between baseline measures of B vitamin status and plasma homocysteine levels to determine the relationship between them. Additional Spearman's Rho correlations were run between B vitamin status and homocysteine change scores. No adjustments were made for multiple comparisons. Vitamin B12 and folate 1evels, plasma homocysteine and global cognitive status were compared between time points. Plasma homocysteine scores were logarithmically transformed. For non-parametric data, a Wilcoxon Signed Rank test was used, and for parametric data, a Paired-sample t-test was used.

The statistical significance threshold was set at $p<0.05$. All statistical analyses were performed using SPSS (IBM SPSS statistics V.21; SPSS Inc, Chicago, Illinois, USA).

\section{Results}




\subsection{Demographics}

Thirty-eight participants with a diagnosis of $\mathrm{AD}$ met the inclusion criteria and were tested at baseline and on average 389.4 days later $(\mathrm{SD}=26.2)$. Participants mean age was 81.3 years $(\mathrm{SD}=6.0)$, had a mild to moderate diagnosis at baseline, and were all free from major depression $(\mathrm{CSDD} \leq 10)$. See Table 1 for full participant demographics.

-----Table 1 Here -----

\subsection{Validation of dietary intake vs plasma levels of $B$ vitamins}

Dietary intake of vitamin B12 did not significantly correlate with plasma vitamin B12 concentrations at baseline $\left(\mathrm{r}_{\mathrm{s}}=.12, \mathrm{p}=.46\right)$ or at follow-up $\left(\mathrm{r}_{\mathrm{s}}=-.18, \mathrm{p}=.29\right)$. Dietary intake of folate did however significantly correlate with plasma folate at baseline $\left(\mathrm{r}_{\mathrm{s}}=.43, \mathrm{p}<.01\right)$ but not at follow-up $\left(\mathrm{r}_{\mathrm{s}}=.06, \mathrm{p}=.72\right)$.

\subsection{The relationship between plasma homocysteine concentrations and vitamin B12 and} folate status

Baseline plasma homocysteine concentrations significantly correlated with plasma folate $\left(\mathrm{r}_{\mathrm{s}}=\right.$ $-.58, \mathrm{p}<.001)$ and vitamin B12 concentrations $\left(\mathrm{r}_{\mathrm{s}}=-.42, \mathrm{p}<.01\right)$. Baseline plasma homocysteine concentrations also significantly correlated with dietary intake of folate $\left(\mathrm{r}_{\mathrm{s}}=-\right.$ $.34, p=.04)$, but not with dietary intake of vitamin B12 $\left(\mathrm{r}_{\mathrm{s}}=-.20, \mathrm{p}=.24\right)$. See Table 2 . 


\subsection{Longitudinal changes in dietary intake and plasma levels}

Dietary and plasma levels of vitamin B12 and folate declined between time-points. However, we only observed borderline significant decrease in plasma B12 concentrations $(p=.05)$. Interestingly, plasma homocysteine concentrations significantly increased between timepoints $(\mathrm{p}=.001)$ as presented in Table 3 .

-----Table 3 Here -----

\subsection{Relationship between $B$ vitamins and change in homocysteine}

No baseline indices of vitamin B status was significantly associated with longitudinal changes of plasma homocysteine ( $p>0.05$ ) (See Table 2). However, there was a trend toward significance between change in homocysteine compared to change in plasma B12 levels $\left(\mathrm{r}_{\mathrm{s}}=\right.$ $-.30, \mathrm{p}=.07)$. This was not observed when compared to changes in plasma folate levels $\left(\mathrm{r}_{\mathrm{s}}=-\right.$ $.10, \mathrm{p}=.54)$.

\section{Discussion}

The primary aim of this research was to assess whether the longitudinal changes in homocysteine levels were related to B vitamin (vitamin B12 and folate) concentrations in a cohort with established AD. This study found that homocysteine levels did increase over the study period, even in a cohort with high homocysteine levels at baseline. However, we were unable to show that the continued increase in homocysteine levels were driven by changes in B vitamin status. 
It is important to highlight that participants in this study reported consuming well above the Reference Nutritional Intake (RNI; (Department of Health, 1991) for folate (mean $=169.6 \%$ ) and vitamin B12 (mean $=529.3 \%)$ at baseline. Whilst FFQs do tend to overestimate intake, there is an indication in the present sample that patients during the early stages of AD consume nutrients sufficient to meet the requirements of $97.5 \%$ of their non-dementia counterparts. In addition, the corresponding plasma concentrations of folate and vitamin B12 were deemed as being well above deficiency levels (Smith and Refsum, 2012; World Health Organization, 2012). There was also no significant decline in B vitamin intake and plasma concentrations over the study period. In any case, all values were still safely well above recommended levels at the end of the study. In contrast, plasma homocysteine concentrations significantly increased between time-points. Therefore, it can be inferred that maintaining an adequate day-to-day intake of B vitamins, even in the relatively high homocysteine levels reported in this sample, is insufficient to maintain baseline homocysteine levels. Correlation analysis between change in homocysteine scores and indices of B vitamin status revealed that there was no significant association, though a trend was found between change in B12 levels and homocysteine levels $(\mathrm{p}=0.07)$. This raises the question whether other factors may be contributing to increasing homocysteine levels within $\mathrm{AD}$ and indirectly may explain that why clinical trials with established AD has not so far shown clinical benefit. It has previously been recommended that higher B vitamin target concentrations are needed to control homocysteine concentrations in certain populations (Smith and Refsum, 2012) and whilst the present study does not provide direct evidence to support this recommendation, it certainly could explain some of our current findings.

More favourable data has been presented in a MCI cohort where vitamin supplementation not only lowered homocysteine levels but protected against brain atrophy (Smith et al., 2010), and cognitive and clinical decline in those with elevated homocysteine at baseline (de Jager et al., 
2012). It is still worth acknowledging that the role of homocysteine in the progression of AD is likely to be more complex, and may interact with other factors. For example, B vitamin supplementation only slowed brain atrophy rates and cognitive decline in participants with sufficiently high plasma levels of omega-3 fatty acids in a MCI population (Jernerén et al., 2015; Oulhaj et al., 2016). Omega-3 fatty acids were not measured in the present study, and therefore we are unable to comment on the role of omega-3 in the context of the current findings.

This study has several limitations which need to be acknowledged. A key limitation is that the relatively small sample size increases the likelihood of type IIerror. Dietary intake estimates calculated from the FFQ should not be considered as absolute values but rather estimates for the comparison within and between participants at different time-points. The participants recruited for this study were recruited for the purposes to identify the role of lifestyle factors on the cognitive progression of dementia (see Farina et al., 2016), and as a result no control group was recruited. This prevents us from discussing how these vitamins and homocysteine concentrations in our sample differ to a cognitively healthy population, and whether the increase in homocysteine over time reported here differs significantly from what we expect in age-matched controls (Frick et al., 2004; Refsum et al., 2004). However, it is well established that homocysteine levels are elevated in $\mathrm{AD}$ compared to healthy controls (for review see (Zhuo et al., 2011). The mean homocysteine concentration at baseline reported in the present study, $16.0 \mu \mathrm{mol} / \mathrm{L}$, is considered elevated and is not dissimilar to that reported in a similar AD cohort (e.g. mean age $=82.8$ years, average total homocysteine $=18.3 \mu \mathrm{mol} / \mathrm{L})($ Joosten et al., 1997). Another potential limitation is the choice of measurement for B vitamin status in the bloods, which may have affected accuracy. For example, holotranscobalamin (holoTC) has been argued as being more reliable marker of B12 status than total vitamin B12 alone 
(Harrington, 2017), particularly in those who are vitamin B12 deficient (Nexo and HoffmannLücke, 2011).

In summary, $\mathrm{AD}$ patients in the present sample showed stable and adequate levels of folate and vitamin B12 for the duration of the study, based on commonly used recommendations and cutoff values. Despite this, homocysteine levels were elevated at baseline and increased further as the disease progressed. Whilst there is an established relationship between elevated homocysteine and dietary intake of B vitamins in older adults (Selhub et al., 1993), this study could indicate that the recommended guidelines in terms of plasma levels of B vitamins might have to be reconsidered, as suggested by others (Smith and Refsum, 2012). However, we should not neglect the potential of other factors, with evidence from a MCI population identifying omega-3 may contribute to hyperhomocysteinemia (Jernerén et al., 2015; Oulhaj et al., 2016). Overall this might explain why so far dietary vitamin B intervention has not showed direct clinical benefit in AD. Ultimately, understanding the underlying mechanism of hyperhomocystemia in AD beyond vitamin B cofactors might help better optimise treatment strategies, which may result in direct clinical benefit to patients.

\section{Acknowledgements}

This work was financially supported by the UK Economic Social and Research Council $(\mathrm{ES} / 102803 \mathrm{X} / 1)$ for the lead author (NF) to complete his PhD. Thank you to Prof Jennifer Rusted who supervised the NF during his PhD and collecting data. Thank you to Prof Refsum and Prof Smith for the use of their lab, and Cynthia Prendergast for assisting with the vitamin assays. Additional support was received the National Institute of Health Research Clinical Research Network and the Norwegian Research Council. 
The authors would like to acknowledge the contribution of the staff and participants of the EPIC-Norfolk Study in the development of the FFQ. EPIC-Norfolk is supported by the Medical Research Council programme grants (G0401527, G1000143) and Cancer Research UK programme grant (C864/A8257).

A special thanks to all the participants that gave their time to take part in this research which would not have been possible without them. 


\section{References}

Aisen, P.S., Schneider, L.S., Sano, M., Ramon Diaz-Arrastia, Christopher H. van Dyck, Myron F. Weiner, Teodoro Bottiglieri, Shelia Jin, Karen T. Stokes, Ronald G. Thomas, Leon J. Thal, Alzheimer Disease Cooperative Study, 2008. High-dose b vitamin supplementation and cognitive decline in alzheimer disease: A randomized controlled trial. JAMA 300, 17741783. doi:10.1001/jama.300.15.1774

Alexopoulos, G.S., Abrams, R.C., Young, R.C., Shamoian, C.A., 1988. Cornell scale for depression in dementia. Biol. Psychiatry 23, 271-284. doi:10.1016/0006-3223(88)90038-8

Bingham, S.A., Welch, A.A., McTaggart, A., Mulligan, A. a, Runswick, S. a, Luben, R., Oakes, S., Khaw, K.T., Wareham, N., Day, N.E., 2007. Nutritional methods in the European Prospective Investigation of Cancer in Norfolk. Public Health Nutr. 4, 847-858. doi:10.1079/PHN2000102

Blasko, I., Hinterberger, M., Kemmler, G., Jungwirth, S., Krampla, W., Leitha, T., Tragl, K.H., Fischer, P., 2013. Conversion from mild cognitive impairment to dementia: Influence of folic acid and vitamin B12 use in the vita cohort. J. Nutr. Health Aging 16, 687-694. doi:10.1007/s12603-012-0051-y

Casey, D.A., Antimisiaris, D., O’Brien, J., 2010. Drugs for Alzheimer's Disease: Are They Effective? Pharm. Ther. 35, 208-211.

Clarke, R., Smith, A.D., Jobst, K.A., Refsum, H., Sutton, L., Ueland, P.M., 1998. Folate, vitamin B12, and serum total homocysteine levels in confirmed Alzheimer disease. Arch. Neurol. 55, $1449-1455$.

de Jager, C.A., Oulhaj, A., Jacoby, R., Refsum, H., Smith, A.D., 2012. Cognitive and clinical outcomes of homocysteine-lowering B-vitamin treatment in mild cognitive impairment: a randomized controlled trial. Int. J. Geriatr. Psychiatry 27, 592-600. doi:10.1002/gps.2758

Department of Health, 1991. Dietary Reference Values for Food, Energy and Nutrients for the United Kingdom: Report of the Panel on Dietary Reference Values of the Committee on Medical Aspects of Food Policy. H.M. Stationery Office, London.

Farina, N., Tabet, N., Rusted, J., 2016. The relationship between habitual physical activity status and executive function in individuals with Alzheimer's disease: a longitudinal, cross-lagged panel analysis. Aging Neuropsychol. Cogn 23, 234-252. doi:10.1080/13825585.2015.1080213

Frick, B., Schroecksnadel, K., Neurauter, G., Leblhuber, F., Fuchs, D., 2004. Increasing production of homocysteine and neopterin and degradation of tryptophan with older age. Clin. Biochem. 37, 684-687. doi:10.1016/j.clinbiochem.2004.02.007

Harrington, D.J., 2017. Laboratory assessment of vitamin B12 status. J. Clin. Pathol. 70, 168-173. doi:10.1136/jclinpath-2015-203502

Huang, C., Chang, W., Huang, S., Lui, C., Chen, N., Chang, Y., Lee, C., Chang, C., Chang, A.Y.W., 2013. Impact of homocysteine on cortical perfusion and cognitive decline in mild Alzheimer's dementia. Eur. J. Neurol. 20, 1191-1197. doi:10.1111/ene.12159

Huang, C., Chang, W., Lui, C., Chen, C., Lu, C., Wang, Y., Chen, C., Juang, Y., Lin, Y., Tu, M., Chang, C., 2010. Impacts of Hyper-Homocysteinemia and White Matter Hyper-Intensity in Alzheimer's Disease Patients with Normal Creatinine: An MRI-Based Study with Longitudinal Follow-up. Curr. Alzheimer Res. 7, 527-533. doi:10.2174/156720510792231702

Jernerén, F., Elshorbagy, A.K., Oulhaj, A., Smith, S.M., Refsum, H., Smith, A.D., 2015. Brain atrophy in cognitively impaired elderly: the importance of long-chain $\omega-3$ fatty acids and B vitamin status in a randomized controlled trial. Am. J. Clin. Nutr. ajcn103283. doi:10.3945/ajen.114.103283

Joosten, E., Lesaffre, E., Riezler, R., Ghekiere, V., Dereymaeker, L., Pelemans, W., Dejaeger, E., 1997. Is Metabolic Evidence for Vitamin B-12 and Folate Deficiency More Frequent in Elderly Patients With Alzheimer's Disease? J. Gerontol. A. Biol. Sci. Med. Sci. 52A, M76M79. doi:10.1093/gerona/52A.2.M76

Kelleher, B.P., Broin, S.D., 1991. Microbiological assay for vitamin B12 performed in 96-well microtitre plates. J. Clin. Pathol. 44, 592-595. 
Kwok, T., Lee, J., Law, C.B., Pan, P.C., Yung, C.Y., Choi, K.C., Lam, L.C., 2011. A randomized placebo controlled trial of homocysteine lowering to reduce cognitive decline in older demented people. Clin. Nutr. 30, 297-302. doi:10.1016/j.clnu.2010.12.004

Madsen, S.K., Rajagopalan, P., Joshi, S.H., Toga, A.W., Thompson, P.M., 2015. Higher homocysteine associated with thinner cortical gray matter in 803 participants from the Alzheimer's Disease Neuroimaging Initiative. Neurobiol. Aging, Novel Imaging Biomarkers for Alzheimer's Disease and Related Disorders (NIBAD) 36, Supplement 1, S203-S210. doi:10.1016/j.neurobiolaging.2014.01.154

Mioshi, E., Dawson, K., Mitchell, J., Arnold, R., Hodges, J.R., 2006. The Addenbrooke's Cognitive Examination Revised (ACE-R): a brief cognitive test battery for dementia screening. Int. J. Geriatr. Psychiatry 21, 1078-1085. doi:10.1002/gps.1610

Molloy, A.M., Scott, J.M., 1997. Microbiological assay for serum, plasma, and red cell folate using cryopreserved, microtiter plate method. Methods Enzymol. 281, 43-53.

Morris, M.S., 2012. The Role of B Vitamins in Preventing and Treating Cognitive Impairment and Decline. Adv. Nutr. Int. Rev. J. 3, 801-812. doi:10.3945/an.112.002535

Nelson, H.E., 1982. National Adult Reading Test (NART): For the Assessment of Premorbid Intelligence in Patients with Dementia: Test Manual. NFER-Nelson, Windsor, UK.

Nexo, E., Hoffmann-Lücke, E., 2011. Holotranscobalamin, a marker of vitamin B-12 status: analytical aspects and clinical utility12345. Am. J. Clin. Nutr. 94, 359S-365S. doi:10.3945/ajen.111.013458

Oulhaj, A., Jernerén, F., Refsum, H., Smith, A.D., de Jager, C.A., 2016. Omega-3 Fatty Acid Status Enhances the Prevention of Cognitive Decline by B Vitamins in Mild Cognitive Impairment. J. Alzheimers Dis. 50, 547-557. doi:10.3233/JAD-150777

Oulhaj, A., Refsum, H., Beaumont, H., Williams, J., King, E., Jacoby, R., Smith, A.D., 2010. Homocysteine as a predictor of cognitive decline in Alzheimer's disease. Int. J. Geriatr. Psychiatry 25, 82-90. doi:10.1002/gps.2303

Refsum, H., Smith, A.D., Ueland, P.M., Nexo, E., Clarke,R., McPartlin, J., Johnston, C., Engbaek, F., Schneede, J., McPartlin, C., Scott, J.M., 2004. Facts and Recommendations about Total Homocysteine Determinations: An Expert Opinion. Clin. Chem. 50, 3-32. doi:10.1373/clinchem.2003.021634

Selhub, J., Jacques, P.F., Wilson, P., Rush, D., Rosenberg, I.H., 1993. VItamin status and intake as primary determinants of homocystememia in an elderly population. JAMA 270, 2693-2698. doi:10.1001/jama.1993.03510220049033

Small, B.J., Herlitz, A., Fratiglioni, L., Almkvist, O., Bäckman, L., 1997a. Cognitive predictors of incident Alzheimer's disease: A prospective longitudinal study. Neuropsychology 11, 413420. doi:10.1037/0894-4105.11.3.413

Small, B.J., Viitanen, M., Winblad, B., Bäckman, L., 1997b. Cognitive changes in very old persons with dementia: The influence of demographic, psychometric, and biological variables. J. Clin. Exp. Neuropsychol. 19, 245-260. doi:10.1080/01688639708403855

Smith, A.D., Refsum, H., 2012. Do we need to reconsider the desirable blood level of vitamin B12? J. Intern. Med. 271, 179-182. doi:10.1111/j.1365-2796.2011.02485.x

Smith, A.D., Smith, S.M., de Jager, C.A., Whitbread, P., Johnston, C., Agacinski, G., Oulhaj, A., Bradley, K.M., Jacoby, R., Refsum, H., 2010. Homocysteine-Lowering by B Vitamins Slows the Rate of Accelerated Brain Atrophy in Mild Cognitive Impairment: A Randomized Controlled Trial. PLoS ONE 5, e12244. doi:10.1371/journal.pone.0012244

Sun, Y., Lu, C.-J., Chien, K.-L., Chen, S.-T., Chen, R.-C., 2007. Efficacy of Multivitamin Supplementation Containing Vitamins B6 and B12 and Folic Acid as Adjunctive Treatment with a Cholinesterase Inhibitor in Alzheimer's Disease: A 26-Week, Randomized, DoubleBlind, Placebo-Controlled Study in Taiwanese Patients. Clin. Ther. 29, 2204-2214. doi:10.1016/j.clinthera.2007.10.012

Tu, M.-C., Huang, C.-W., Chen, N.-C., Chang, W.-N., Lui, C.-C., Chen, C.-F., Chen, C., Wang, Y.L., Lin, Y.-T., Chang, C.-C., 2010. Hyperhomocysteinemia in Alzheimer dementia patients and cognitive decline after 6 months follow-up period. Acta Neurol. Taiwanica 19, 168-177. 
Vogel, T., Dali-Youcef, N., Kaltenbach, G., Andrès, E., 2009. Homocysteine, vitamin B12, folate and cognitive functions: a systematic and critical review of the literature. Int. J. Clin. Pract. 63, 1061-1067. doi:10.1111/j.1742-1241.2009.02026.x

World Health Organization, 2012. Serum and red blood cell folate concentrations for assessing folate status in populations. World Health Organization, Geneva.

World Health Organization, 1992. The ICD-10 Classification of Mental and Behavioural Disorders: clinical descriptions and diagnostic guidelines, Second. ed. World Health Organization, Geneva.

Zhuo, J.-M., Wang, H., Praticò, D., 2011. Is hyperhomocysteinemia an Alzheimer's disease (AD) risk factor, an AD marker, or neither? Trends Pharmacol. Sci. 32, 562-571. doi:10.1016/j.tips.2011.05.003 
Table 1. Baseline demographic data of AD participants $(n=38)$

$\% \quad$ Mean $\quad$ SD

Age (years)

81.3

6.0

Gender

Male

53

Female

47

Diagnosis

$\mathrm{AD}$

71

Mixed

29

Race

Caucasian

100

Time Since Diagnosis

1.1

1.5

(years)

Premorbid IQ

115.0

8.9

Age leaving full-time

16.1

education (years)

Pulse Pressure (Systolic

75.4

16.8

Pressure - Diastolic

Pressure)

Creatinine $(\mu \mathrm{mol} / \mathrm{l})$

91.9

31.5

Cornel Scale of

2.5

2.7

Depression in Dementia

Body Mass Index

26.2

3.8 
Table 2. Spearman's correlation between ACE-R change scores, baseline dietary B vitamins, baseline plasma B vitamins and baseline plasma homocysteine concentrations.

\begin{tabular}{|c|c|c|c|c|c|c|c|c|c|c|}
\hline & \multicolumn{2}{|c|}{$\begin{array}{l}\text { Baseline Dietary Vitamin } \\
\text { B12 }\end{array}$} & \multicolumn{2}{|c|}{$\begin{array}{l}\text { Baseline Plasma } \\
\text { Folate }\end{array}$} & \multicolumn{2}{|c|}{$\begin{array}{l}\text { Baseline Plasma } \\
\text { Vitamin B12 }\end{array}$} & \multicolumn{2}{|c|}{$\begin{array}{l}\text { Baseline Plasma } \\
\text { Homocysteine }\end{array}$} & \multicolumn{2}{|c|}{$\begin{array}{c}\text { Plasma Homocysteine } \\
\text { Change scores }\end{array}$} \\
\hline & $r_{s}$ & $\mathrm{p}$ & $\mathrm{r}_{\mathrm{s}}$ & $\mathrm{p}$ & $\mathrm{r}_{\mathrm{s}}$ & $\mathrm{p}$ & & $\mathrm{p}$ & $r_{\mathrm{s}}$ & $\mathrm{p}$ \\
\hline $\begin{array}{l}\text { Baseline Dietary } \\
\text { Folate }\end{array}$ & .44 & .006 & .43 & .008 & -.11 & .531 & & .039 & .01 & .972 \\
\hline $\begin{array}{l}\text { Baseline Dietary } \\
\text { Vitamin B12 }\end{array}$ & & & .27 & .100 & .12 & & -.20 & .236 & .11 & .508 \\
\hline $\begin{array}{l}\text { Baseline Plasma } \\
\text { Folate }\end{array}$ & & & & & 26 & & -.58 & $<.001$ & -.21 & .201 \\
\hline $\begin{array}{l}\text { Baseline Plasma } \\
\text { Vitamin B12 }\end{array}$ & & & & & & & -.42 & .009 & 0.08 & .643 \\
\hline
\end{tabular}


Table 3. The means (and standard deviations) of blood and dietary markers of B vitamins, plasma homocysteine and global cognition at each time-point for participants that completed both time points $(n=38)$.

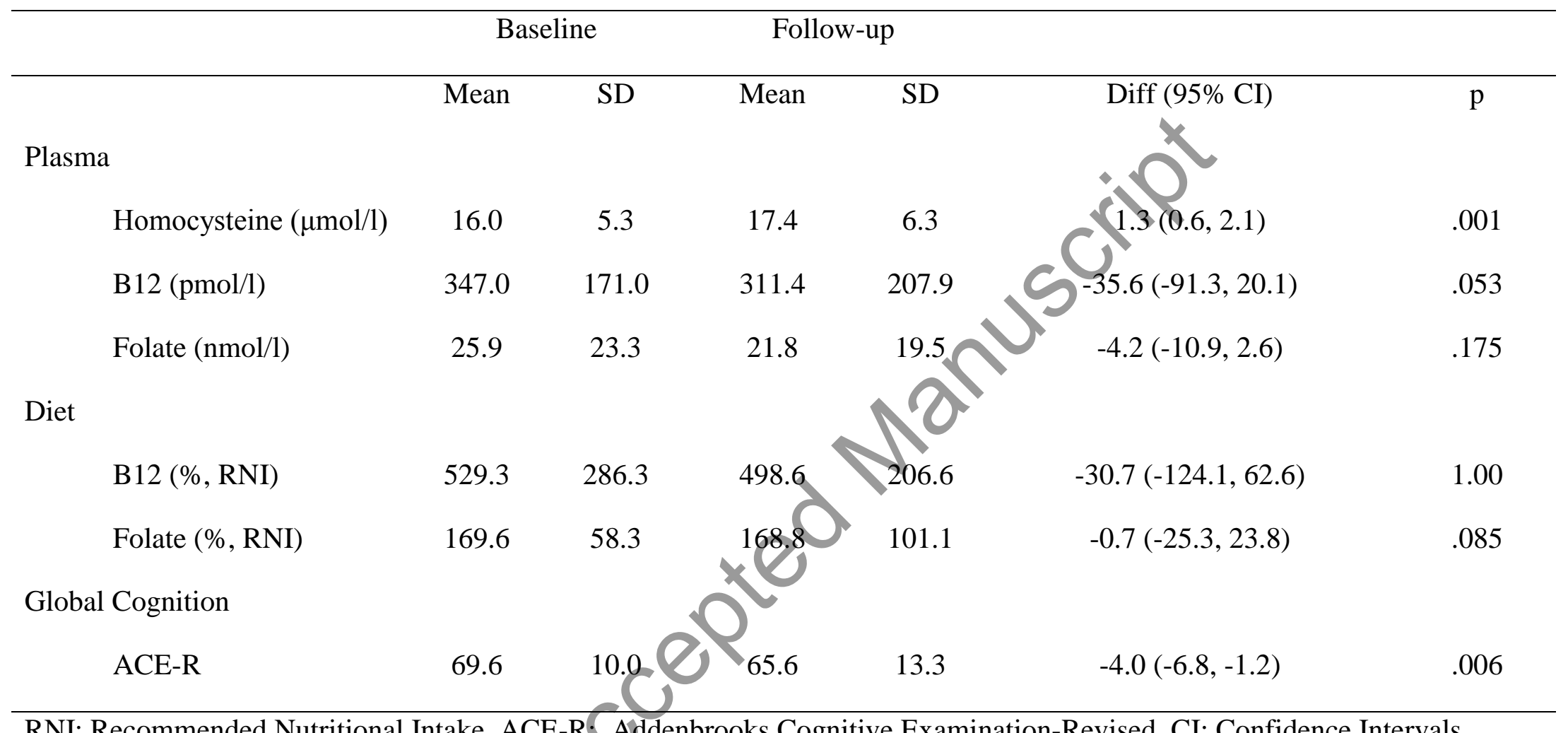

RNI: Recommended Nutritional Intake, ACE-R: Addenbrooks Cognitive Examination-Revised, CI: Confidence Intervals 